\title{
First description of an acquired internal hernia: a case report
}

\author{
Jan Philipp Ramspott · Tarkan Jäger (D) K Klaus Emmanuel · Philipp Schredl
}

Received: 24 February 2019 / Accepted: 8 April 2019 / Published online: 26 April 2019

(c) The Author(s) 2019

\begin{abstract}
Summary
Background An internal hernia is defined as a protrusion from intestines or other abdominal organs through a congenital or acquired aperture within the peritoneal cavity. Internal hernia are rare, with an incidence of less than $1 \%$. Mostly, they are located paraduodenal (Treitz hernia) and show mortality rates up to $50 \%$.

Case presentation We describe the case of a 26-yearold man presenting with acute bowel obstruction and a history of surgical treatment for a congenital diaphragmatic hernia.

Methods Clinical examination and computed tomography (CT) scan were interpreted as intestinal obstruction. Exploratory laparotomy resulting in an appendectomy, small intestine resection, and lysis of adhesions was performed.

Results In situ, an atypical appendix fused to the spleen was detected, forming an aperture within the peritoneal cavity with protrusion and strangulation of the small intestine. The small intestine specimen sent to pathology confirmed necrotic small intestine mucosa.

Conclusion We present the first description of a young man with an internal hernia due to an intraperitoneal aperture formed by his appendix fused to the spleen.
\end{abstract}

Author contributions J.P. Ramspott, T. Jäger, K. Emmanuel, and P. Schredl contributed equally to manuscript writing and critical revision.

J. P. Ramspott · T. Jäger, MD $(\bowtie) \cdot$ K. Emmanuel $\cdot$ P. Schredl Department of Surgery, Salzburger Landeskliniken (SALK), Paracelsus Medical University, Müllner Hauptstraße 48, 5020 Salzburg, Austria ta.jaeger@salk.at

J. P. Ramspott Faculty of Medicine, University Witten/Herdecke, Witten, Germany
Internal hernia are difficult to diagnose but should be included in the differential diagnosis in cases of intestinal obstruction. CT should be performed in cases of suspicion. Surgical intervention shouldn't be delayed in order to reduce the high morbidity and mortality rates.

Keywords Appendix - Bochdalek hernia .

Postoperative complications $\cdot$ Rare disease $\cdot$ Ileus

\section{Main novel aspects}

- We describe the first case of an acquired internal hernia in a young patient presenting with acute intestinal obstruction.

- The patient had had surgeries for a Bochdalek hernia in the past.

- Only exploratory laparotomy revealed that parts of his small intestine herniated through an aperture formed by his appendix fused to the spleen.

\section{Background}

An internal hernia is defined as a protrusion from the intestines or other abdominal organs through a congenital (for example omental foramen) or acquired (post-surgery, post-traumatic, post-infection) aperture within the peritoneal cavity. Internal hernia are rare, with an incidence of less than of $1 \%$. Mostly, they are located paraduodenal (Treitz hernia) [1-3]. $0.6-5.8 \%$ of all small intestine obstructions are caused by internal hernia, with mortality rates of $50 \%$ [2, 4, 5]. We describe the case of a young man presenting with acute intestinal obstruction attributed later to a very rare type of an acquired internal hernia on exploratory laparotomy, which has not been described so far. 


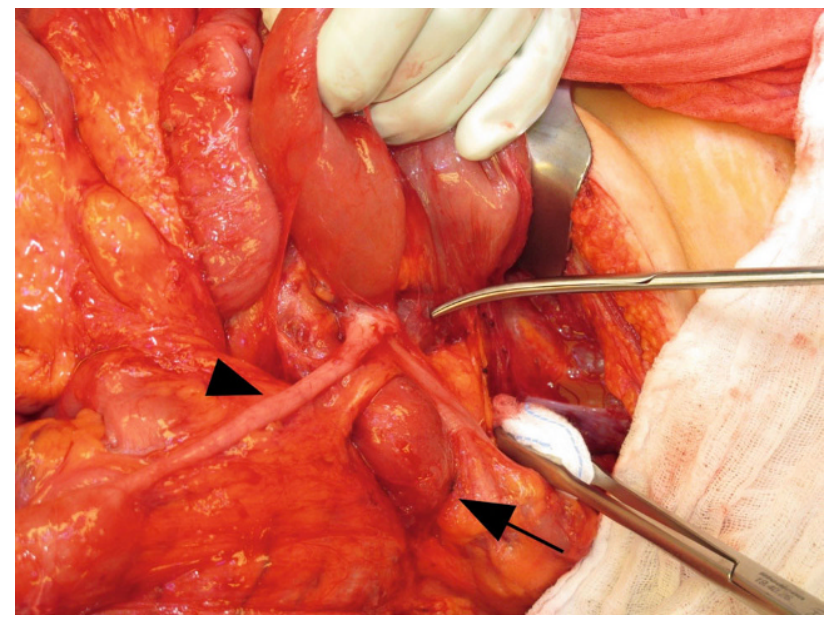

Fig. 1 Intraoperative situs: Fusion from the appendix (arrowhead) and spleen (upper instrument) with protrusion of the small intestine (arrow)

\section{Case presentation}

A 26-year-old male presented to our emergency department with mainly left-sided abdominal pain, nausea, and vomiting. He did not report any fever, dysuria, or change in bowel habits. His past surgical history was remarkable for a congenital diaphragmatic hernia (Bochdalek hernia) with a dystopia of intraabdominal organs (stomach, spleen, and parts of his small and large intestine) in his thoracic cavity. Therefore, he underwent emergent surgery after birth with reposition of his organs and closure of pleura and peritoneum. After one month he relapsed and was treated with a latissimus dorsi flap. On physical examination, abdominal tenderness without any resistance was detected. His laboratory results showed elevated white blood cells (WBC) $15.5 \times 10^{9} / 1$ WBC (reference value $3.5-9.8 \times 10^{9} / 1$ ). We then performed ultrasound, abdominal X-ray, and CT scan, which detected a mechanical small intestine obstruction with $4 \mathrm{~cm}$ dilated small intestine loops and distal bowel collapse. Furthermore, the spleen was dislocated to ventral-caudal.

The patient underwent an exploratory laparotomy due to notable adhesions of the small intestine after Bochdalek hernia repair in the past. The intraoperative surprise was that of an atypical appendix, which had a total length of $15 \mathrm{~cm}$ and was fused to the spleen. In total, the appendix, medial sigmoid colon, and dorsal trunk formed an aperture within the peritoneal cavity causing protrusion and strangulation of the small intestine (Figs. 1 and 2). We performed a classical open appendectomy and resection of $40 \mathrm{~cm}$ chronically altered small intestine followed by a side-to-side anastomosis of the jejunum $100 \mathrm{~cm}$ distal from the duodenojejunal flexure. The histological examination confirmed necrotic small intestine mucosa. Postoperative recovery was delayed by a per-

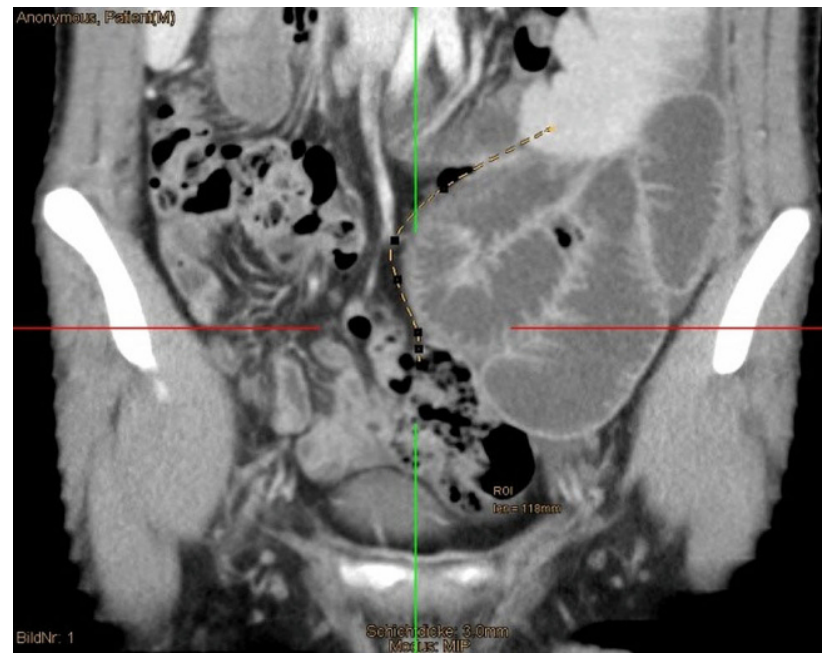

Fig. 2 Maximum intensity projection (MIP) from a venous CT scan with the appendix's planning path (dashed yellow line) from the medial caecum to the distal spleen. Dilated small intestine bowel loops can be detected in the left lower abdomen

sistent atony solved by prokinetic methods. The patient was discharged on the twelfth postoperative day.

\section{Discussion}

An internal hernia is defined as a protrusion from intestines or other abdominal organs through an aperture within the peritoneal cavity which can be congenital or acquired. In decreasing order and depending on their location, they can be grouped as follows: paraduodenal hernia (Treitz, 53\%), pericecal hernia (Rieux, 13\%), hernia through the foramen of Winslow (8\%), transmesenteric (Petersen) and transmesocolic hernia $(8 \%)$, intersigmoid hernia $(6 \%)$, and retroanastomotic hernia (5\%). Paravesical hernia represent $7 \%$ of all internal hernia, even if they are not considered as internal hernia in the broadest sense [1,2].

Internal hernia are rare, but transmesenteric, transmesocolic, and retroanastomotic hernia are increasing due to more and more Roux loops, especially in bariatric surgery $[1,3,5]$.

In adults, previous abdominal surgery can lead to internal herniation by creating new intra-abdominal spaces. Our patient underwent abdominal surgery as a little child, with reposition of his intraabdominal organs suffering from congenital diaphragmatic hernia which could have caused his special pathology.

Clinical presentation of an internal hernia is nonspecific. They can be asymptomatic as well as presenting as a colic-type intestinal cramp with or without nausea or vomiting. In any case of suspicion, a CT scan should be performed [4]. In most cases, symptomatic internal hernia have to be treated surgically [6]. No case of an internal hernia caused by an atypical appendix fused to the spleen and thus forming an aperture within the peritoneal cavity leading to a protrusion of small intestine has been described to date. 


\section{Conclusion}

We report the first description of a young man presenting clinically with an acute intestinal obstruction due to an intraperitoneal aperture formed by a fusion of the appendix to the spleen.

Internal hernia are rare and difficult to diagnose, but they should be included in the differential diagnosis in cases of intestinal obstruction and a history of abdominal surgery. To reduce the high morbidity and mortality rates, surgical intervention shouldn't be delayed.

To our best knowledge, this is the first description of this kind of internal hernia, which is not yet included in the internal hernia classification system.

Funding Open access funding provided by Paracelsus Medical University.

\section{Compliance with ethical guidelines}

Conflict of interest J.P. Ramspott, T. Jäger, K. Emmanuel, and P. Schredl declare that they have no competing interests.

Ethical standards For this article no studies with human participants or animals were performed by any of the authors. Written informed consent was obtained from the patient before writing the case report.
Open Access This article is distributed under the terms of the Creative Commons Attribution 4.0 International License (http://creativecommons.org/licenses/by/4.0/), which permits unrestricted use, distribution, and reproduction in any medium, provided you give appropriate credit to the original author(s) and the source, provide a link to the Creative Commons license, and indicate if changes were made.

\section{References}

1. Meyers MA, Charnsangavej C, Oliphant M. Meyers' dynamic radiology of the abdomen normal and pathologic anatomy. 2011.

2. Gore RM, Levine MS. Textbook of gastrointestinal radiology. Philadelphia: W.B. Saunders; 2000.

3. Martin LC, Merkle EM, Thompson WM. Review of internal hernias: radiographic and clinical findings. Ajr Am J Roentgenol. 2006;186(3):703-17.

4. Akyildiz $\mathrm{H}$, et al. Internal hernia: complex diagnostic and therapeutic problem. IntJ Surg. 2009;7(4):334-7.

5. Karcz WK, et al. Internal hernias after bariatric surgery. Chirurg. 2015;86(9):855-60.

6. Edwards HM, Al-Tayar H. A transmesenteric congenital internal hernia presenting in an adult. J Surg Case Rep. 2013;2013(12):rjt099.

Publisher's Note Springer Nature remains neutral with regard to jurisdictional claims in published maps and institutional affiliations. 